Original Research Paper

\title{
Comparing the Empirical and the Theoretical Probability Density of Return in which Variance Obeying Ornstein- Uhlenbeck Process at Indonesian Stock Exchange IDX
}

\author{
${ }^{1}$ Dwi Satya Palupi, ${ }^{2}$ Eduardus Tandelilin, ${ }^{1}$ Arief Hermanto and ${ }^{1}$ Muhammad Farchani Rosyid \\ ${ }^{1}$ Departemen Fisika, Universitas Gadjah Mada, Yogyakarta, Indonesia \\ ${ }^{2}$ Departemen Manajemen, Universitas Gadjah Mada, Yogyakarta, Indonesia
}

\author{
Article history \\ Received: 23-08-2016 \\ Revised: 31-03-2017 \\ Accepted: 12-05-2017 \\ Corresponding Author: \\ Dwi Satya Palupi \\ Departemen Fisika, Universitas \\ Gadjah Mada, Yogyakarta, \\ Indonesia \\ Email:dwi_sp@ugm.ac.id
}

\begin{abstract}
The distribution of the probability density of a return index with stochastic volatility has been calculated. Here the stock index is assumed to follow geometric Brownian motion, while the variance is assumed to obey Ornstein-Uhlenbeck process as in Heston model. The distribution of the probability density of the return which is obtained by solving the FokkerPlanck equation of two dimensional index and the variance have been compared with the probability density taken from Indonesian Stock Exchange (IDX). In this study, we use Jakarta Islamic Index (JII), LQ45 and Jakarta Composite Index (JCI) data series from 2004 to 2012. We have shown that the theoretical probability density of return obtained from the calculation is in agreement with the empirical probability density. The theoretical probability density with stochastic volatility is closer to the empirical one than that of the Gaussian, particularly at the tail. The variance probability density at stationary state can be obtained by fitting the empirical probability density obtained from IDX data series with an integral expression obtained from quantum mechanical method.
\end{abstract}

Keywords: Return, IDX, Variance, Stochastic Volatility

\section{Introduction}

The behaviour of a financial market is very interesting not only for economists but also for physicists and mathematicians. The financial market has abundant data that challenges physicists as well as mathematicians to give a suitable explanation. The application of the stochastic process in economics, especially in finance dates back to 1900 when Bachelier proposed a model in which stock price was assumed to have Gaussian distribution (Mantegna and Stanley, 2000). In 1963, Mandelbrot proposed another model of stock prices in which the stock price was assumed to obey log-normal distribution (Mandelbrot, 1963). One decade later, Black and Scholes (1973) proposed a stochastic model describing option price. He assumed that the option price follows a process called geometric Brownian motion with constant volatility (Black and Scholes, 1973). However, more careful studies on financial data series showed that the distribution associated with stock price is non-Gaussian where the tail of the probability density is fatter, i.e., decreases slower than that of the Gaussian distribution. Moreover, the tail of the probability density associated with stock price decreases as slow as a power law. The careful studies of the shape of the distribution density of stock price have been made recently by several scientists to understand the behaviour of financial data series (Nava et al., 2016; Carranco et al., 2016; Guhathakurta et al., 2006; Huang et al., 2003; Yamasaki et al., 2005). One of the proposed models to explain the fatness of the tail of the probability density associated with stock price assumes that the volatility of the distribution follows Ornstein-Uhlenbeck stochastic process (Hull and White, 1987; Stein and Stein, 1991; Heston, 1973; Belal, 2004).

This work is the sequel to our former work (Palupi et al., 2014) and is in line with the work of (Dragulescu and Yakovenko, 2002). In this study, the stock price under consideration is assumed to follow geometric Brownian motion and the variance (the square of volatility) is assumed to follow Ornstein-Uhlenbeck process as in Heston model (Heston, 1993). The calculation of the probability density is carried out by involving two-dimensional Fokker-Planck equation for logreturn and solved by using the path integral method as already discussed deeply in (Palupi et al., 2014). In this 
study, we compare the theoretic probability density with the probability density calculated from the data of IDX. Here we point out that the theoretic probability density is in agreement with the empirical probability density obtained from IDX data series. The variance of the stock price in IDX follows Ornstein-Uhlenbeck process. The variance probability density at stationary state can be obtained by fitting the empirical probability density obtained from IDX data series with an integral expression obtained from the quantum mechanical method.

\section{Materials and Methods}

Financial market can be considered as a manyparticle system in which stocks are regarded as particles and stock prices as the positions of particles in a price space. Stock particles move randomly as diffusion process obeying the stochastic differential:

$d S(t)=\phi S(t) d t+\sigma S(t) d W_{S}$

Where:

$S=$ Particles position (stock price)

$\phi \quad=$ A parameter

$d W_{s}=$ The Wiener standard process for the stock price

$\sigma=$ The volatility of stock price

Equation 1 is stochastic differential equation for Brownian Geometric process, while the variance $v$ (and the volatility, $v=\sigma^{2}$ ) obeys Ornstein-Uhlenbeck process described by the following stochastic differential equation:

$$
d v(t)=-\mu(v-\eta) d t+\kappa \sqrt{v} d W_{v}
$$

where, the variance reaches $\eta$ at a long time with speed $\mu, \kappa$ is the volatility of the stock price variance $v, d W_{v}$ is Wiener standard process for the variance $v$.

The variance behaves as friction trying or tending to reverse to the average of variance.

According to Ito's formula of stochastic differential equation, if $X(t)$ is an Ito stochastic process then $X(t)$ satisfies $d X(t)=f(t) d t+g(t) d W(t)$ (Kuo, 2006). Futher more when $Y$ is a stochastic process depending on $X(t)$, i.e., $Y=Y(t, X(t))$, then $Y$ obeys the following stochastic differential equation $d Y(t, X(t))=\left(\frac{\partial Y}{\partial t}+\frac{\partial Y}{\partial x} f(t)+\frac{1}{2} \frac{\partial^{2} Y}{\partial x^{2}} g(t)\right) d t+\frac{\partial Y}{\partial x} g(t) d W(t)$ (Kuo, 2006).

With the change of variable $z=l_{r}-\phi t$ and $l_{r}=$ In $(S(t) / S(0))$ Ito's formula, Equation 1 can be written as: $d z=-\frac{v}{2} d t+\sqrt{v} d W_{S}$

where, $z$ is $\log$ natural-return relative to the parameter $\phi$.

Since particles of stock follow a diffusion process, the probability density equation of return associated to Equation 1 and 3 satisfies:

$$
\begin{aligned}
& \frac{\partial P}{\partial t}=\mu \frac{\partial}{\partial v}[(v-\eta) P]+\frac{1}{2} \frac{\partial}{\partial z}(v P) \\
& +\rho \kappa \frac{\partial^{2}}{\partial z \partial v}(v P)+\frac{1}{2} \frac{\partial^{2}}{\partial z^{2}}(v P)+\frac{\kappa}{2} \frac{\partial^{2}}{\partial v^{2}}(v P)
\end{aligned}
$$

The detail of the derivation of Equation 4 can be found in (Kuo, 2006). Equation 4 is referred to as two-dimensional Fokker-Planck equation, where $P=$ $P\left(z, v \mid v_{i}\right)$ is the probability density of the transition from initial state at $z=0$ with variance $v_{i}$ to jump to state at arbitrary $z$ with variance $v$. Equation 4 can be converted to the following Schroedinger-like equation as in quantum mechanics:

$\frac{\partial \tilde{P}\left(v \mid v_{i}\right)}{\partial t}=-\hat{H} \tilde{P}\left(v \mid v_{i}\right)$

where:

$$
\begin{aligned}
& \hat{H}=\frac{\kappa^{2}}{2} \hat{p}_{v}^{2} \hat{v}-i \mu \hat{p}_{v}(\hat{v}-\eta)+\frac{p_{z}^{2}-i p_{z}}{2} \hat{v} \\
& +i \rho \kappa p_{z} \hat{p}_{v} \hat{v}
\end{aligned}
$$

And $\hat{p}_{v}=-i d / d v$ is momentum operator being a canonic conjugate of $\hat{v}$ and satisfying commutation relation $\left[\hat{v}, \hat{p}_{v}\right]=i$ and $\tilde{P}=\tilde{P}\left(v, t \mid v_{i}, t_{i}\right)$ is the probability density of transition from the initial state at $t=t_{i}$ with variance $v_{i}$ to final state with variance $v$ at $t$. The solution of Equation 6 is written as:

$$
\tilde{P}\left(v, t \mid v_{i}, t_{i}\right)=\left\langle v_{f}|\exp (-\hat{H} t)| v_{i}\right\rangle
$$

The path integral formulation for the return probability density in Equation 7 is given by:

$$
\tilde{P}\left(v, t \mid v_{i}, t_{i}\right)=\int \mathrm{D} v \mathrm{D} p_{v} e^{S}
$$

where, the action $S$ is given by: 


$$
\begin{aligned}
& S=\mathrm{i} p_{v M} v_{M}-\mathrm{i} p_{v i} v_{i}+ \\
& \sum_{j=1}^{M-1} v_{j}\left[\begin{array}{l}
\mathrm{i}\left(p_{v j-1}-p_{v j}\right)-\varepsilon \frac{\kappa^{2}}{2} p_{v j}^{2}+\mathrm{i} \varepsilon \mu p_{v j} \\
-\varepsilon \frac{p_{z}^{2}-\mathrm{i} p_{z}}{2}-\in \rho \kappa p_{z} p_{v j}
\end{array}\right] \\
& -\sum_{j=1}^{M} \mathrm{i} \varepsilon \mu p_{v j} \eta
\end{aligned}
$$

Integrating Equation 8 yields:

$$
\begin{aligned}
& P\left(z \mid v_{i}\right)=\int_{-\infty}^{\infty} \frac{d p_{z} e^{\mathrm{i} p_{z} z}}{(2 \pi)} \exp \left[\frac{-\left(p_{x}^{2}-\mathrm{i} p_{x}\right) v_{i}}{\Gamma+\omega \operatorname{coth}\left(\frac{\omega t}{2}\right)}\right] \\
& \times \exp \left[-\frac{2 \mu \eta}{\kappa^{2}} \ln \frac{\left(\Gamma \sinh \left(\frac{\omega t}{2}\right)+\omega \cosh \left(\frac{\omega t}{2}\right)\right)}{\omega}+\frac{\mu \eta \Gamma t}{\kappa^{2}}\right]
\end{aligned}
$$

where, $\chi=\mu+i \rho \kappa p_{z}$ and $\omega=\sqrt{\chi^{2}+\kappa^{2}\left(p_{z}^{2}-i p_{z}\right)}$. The variance or volatility does not appear explicitly from data series. The probability density of the variance at stationary state can be obtained from Equation 2 as:

$$
\Pi_{*}\left(v_{i}\right)=\frac{\alpha^{\beta+1}}{\Gamma(\beta+1)} v_{i}^{\beta} \exp \left(-\alpha v_{i}\right)
$$

where, $\alpha=2 \mu / \kappa^{2}$ and $\beta=\alpha \eta-1, \Pi *\left(\mathrm{v}_{\mathrm{i}}\right)$ probability density of variance at sationary state $\Gamma$ gamma function.

If we assumed that the probability density of volatility is stationer, the probability density that the value of $\log$ return is $z$ for every value of volatility is given by:

$$
\begin{aligned}
& P(z)=\frac{1}{(2 \pi)} \int_{-\infty}^{\infty} d p_{z} e^{i p_{z} z} \\
& \times \exp \left(\begin{array}{l}
\frac{\mu \eta \Gamma t}{\kappa^{2}}-\frac{2 \mu \eta}{\kappa^{2}} \\
\ln \left(\frac{\left(\omega^{2}-\Gamma^{2}\right)+\Gamma 2 \mu}{2 \omega \mu} \sinh \frac{\omega t}{2}+\cosh \left(\frac{\omega t}{2}\right)\right)
\end{array}\right)
\end{aligned}
$$

\section{Results and Discussion}

The parameter $\mu, \eta$ and $\kappa$ in Equation 1 and Equation 2 can be determined by fitting the empirical probability density obtained from IDX data series with Equation 12 . We use Jakarta Composite Index (JCI), LQ45 and Jakarta Islamic Index (JII) data series in time interval 2004-2012 in which every index contains 2187 points data. Each point of data is the stock price at closing. The data series of the stock price at closing is shown Fig. 1a shows JCI data series and Fig. 1b shows data series for LQ45 and JII.
Figure $1 \mathrm{a}$ and $1 \mathrm{~b}$ show that the JCI index has higher values than that of LQ45 and JII. However, JCI, LQ45 and JII data series have the same shape of fluctuation. The values of the indexes are different because the indexes have different initial values.

The return probability density for each index is shown respectively in Fig. 2-4. On every figure, the solid line represents the theoretical probability density which is calculated from Equation 12, whereas the dots represent the empirical probability density. The values of $\mu, \eta$ and $\kappa$ which are obtained from the fitting are presented in Table 1.

Figure 2-4 show that the theoretical probability densities are in agreement with the empirical probability density obtained from LQ45, JII and JCI data series at IDX. So the assumption that variance is not constant but changes stochastically is seemingly acceptable for data series at IDX. Since stock price and the associated variance respect a two-dimensional diffusion process, it permits us to construct a Fokker-Planck equation governing the probability density for the diffusion process. The Fokker-Planck equation must be solved in order to get the return probability density for stock price as well as variance. The Fokker-Planck equation describes the dynamics or time evolution of the return probability density, while Schroedinger equation determines the time evolution of the quantum mechanical state of a system, i.e., the time evolution of probability amplitude from which we obtain the time evolution of the probability density. In this study, we solve the equation by making use of the quantum mechanical method described in (Palupi et al., 2014). The solution is given as integral in Equation 12. To calculate the integral numerically we need the values of parameters given in Table 1 .

From Fig. 2-4, we can see that the theoretical return probability density with stochastic variance is closer to the empirical one than that of the Gaussian (with constant volatility). Both the theoretic and the empirical return probability density have Gaussian distribution at peak, but the tail of both probability density is fatter than that of the Gaussian. The tail of JCI return probability density is shown in Fig. 2b and. 2c, the tail of LQ45 return probability is shown in Fig. $3 \mathrm{~b}$ and $3 \mathrm{c}$ and that of JII is shown in Fig. $4 \mathrm{~b}$ and $4 \mathrm{c}$. The tail of each return probability density is closer to that of our theoretical return probability density than that of the Gaussian distribution.

In Fig. 2-4, we can see that the empirical (and the theoretical) return probability densities are not symmetric as in the Gaussian distribution, so we can conclude that this stochastic variance model is more comparable to data series than that of the Gaussian.

Table 1 . The value of parameter constants

\begin{tabular}{llll}
\hline & $\mu(/$ day $)$ & $\eta(/$ day $)$ & $\kappa(/$ day $)$ \\
\hline JCI & 0.00090 & 0.00056 & 0.00013 \\
LQ45 & 0.00051 & 0.00082 & 0.00010 \\
JII & 0.00200 & 0.00076 & 0.00035 \\
\hline
\end{tabular}




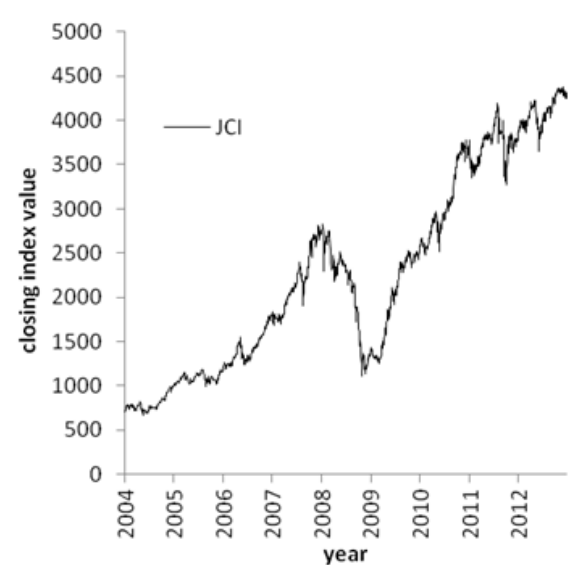

(a)

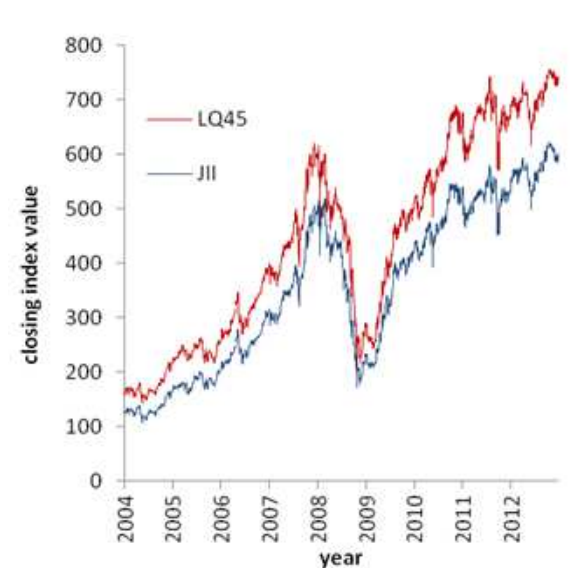

(b)

Fig. 1. JCI, LQ45 and JII time evolution at closing day

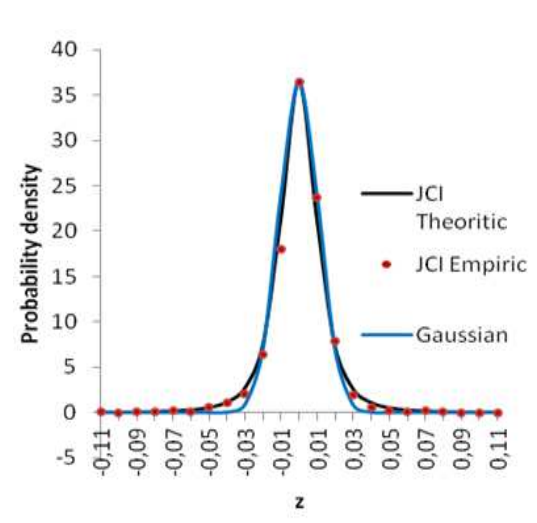

(a)

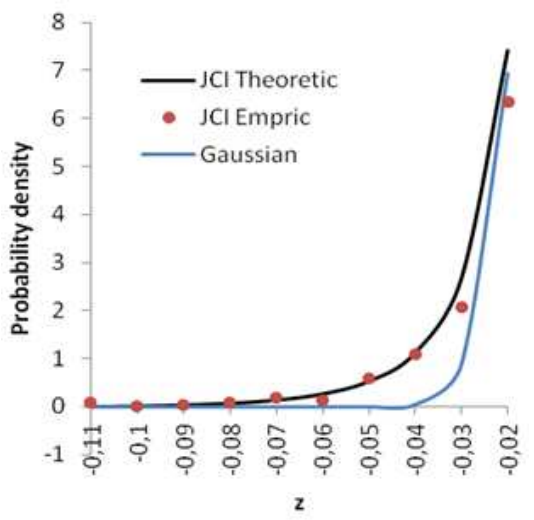

(b)

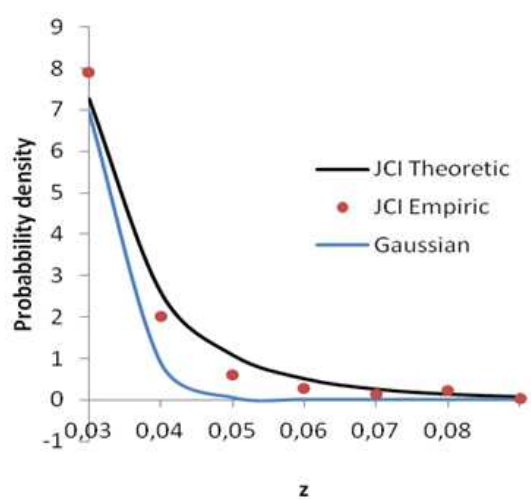

(c)

Fig. 2. Probability density of JCI. The dots are empiric probability and the black solid is theoretic probability and the blue is Gaussian (a) full probability density of JCI (b) the left tail of probability density (c) the right tail of probability density. The theoretical probability density with stochastic volatility is closer to the empirical one than that of the Gaussian, especially at the tail probability density

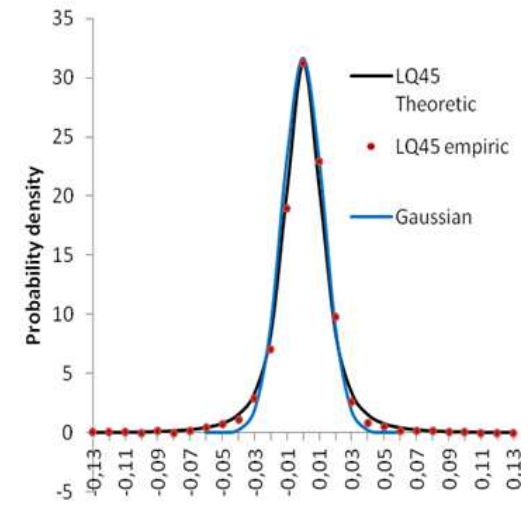

(a)

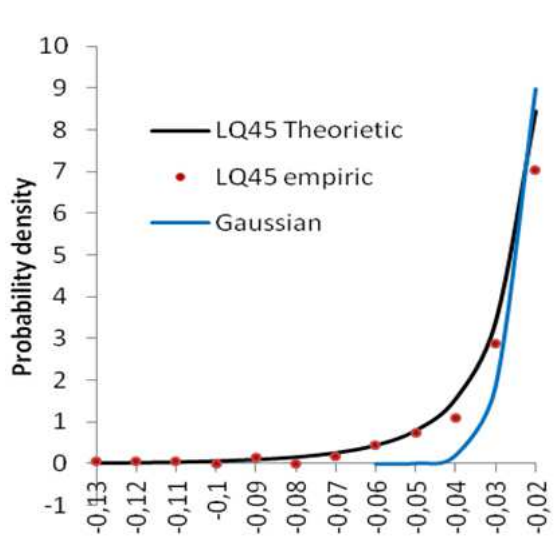

(b)

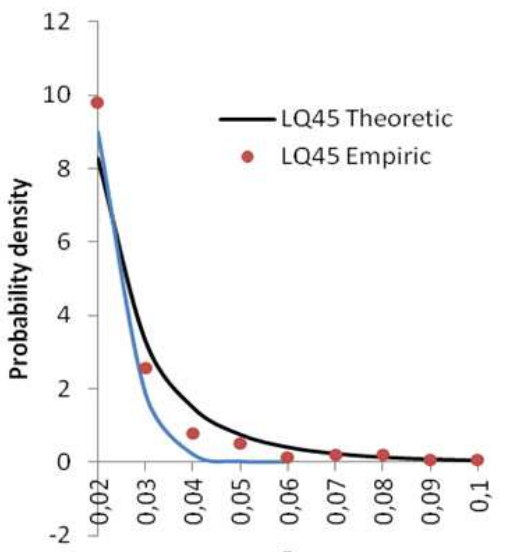

(c)

Fig. 3. Probability density of LQ45. The dots are empiric probability and the black solid is theoretic probability and the blue is Gaussian (a) Full probability density of LQ45 (b) The left tail of probability density (c) the right tail of probability density. As at JCI, the theoretic probability density with stochastic volatility is closer to the empirical one than that of the Gaussian, particularly at the tail probability density 


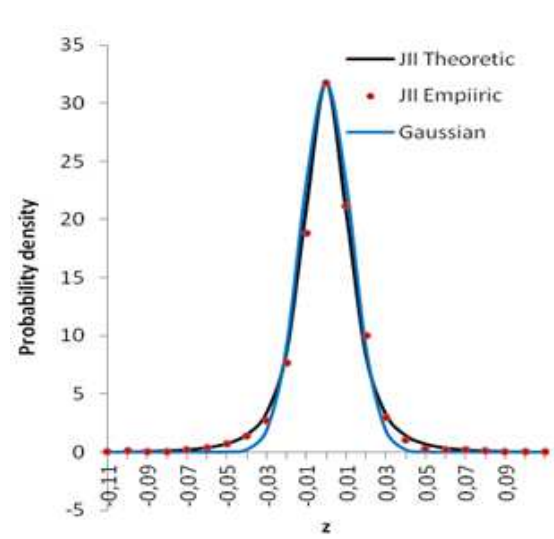

(a)

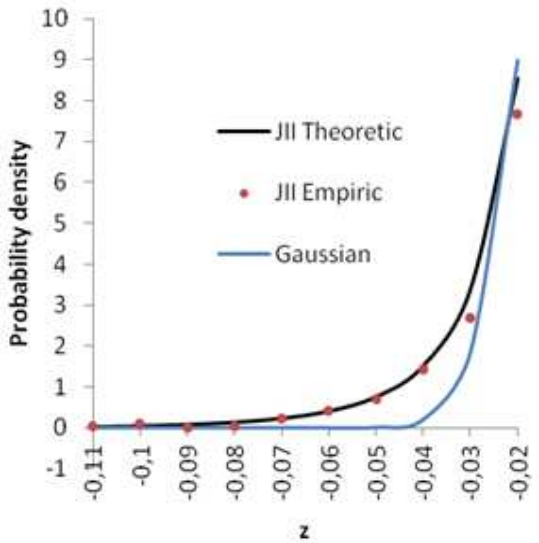

(b)

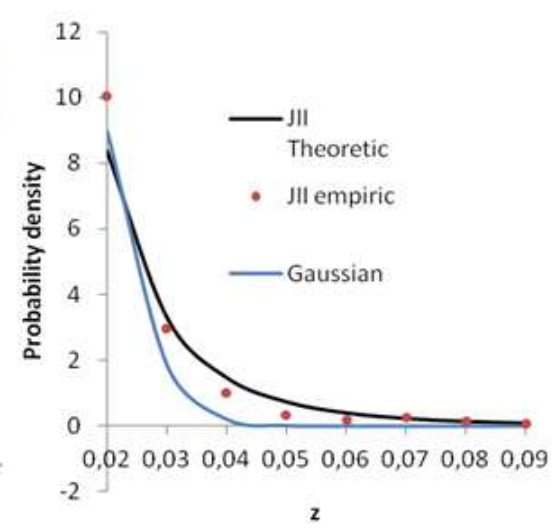

(c)

Fig. 4. Probability density of JII. The dots are empiric probability and the black solid is theoretic probability and the blue is Gaussian (a) full probability density of JII (b) the left tail of probability density (c) the right tail of probability density. As at JCI and LQ45, the theoretic probability density with the stochastic volatility of JII is closer to the empirical one than that of the Gaussian, particularly at the tail probability density

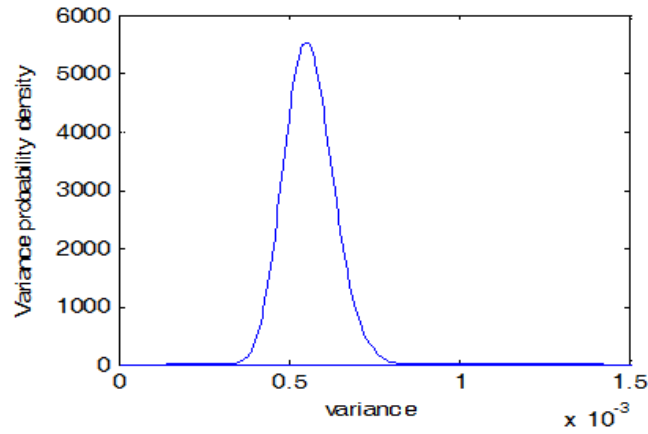

(a)

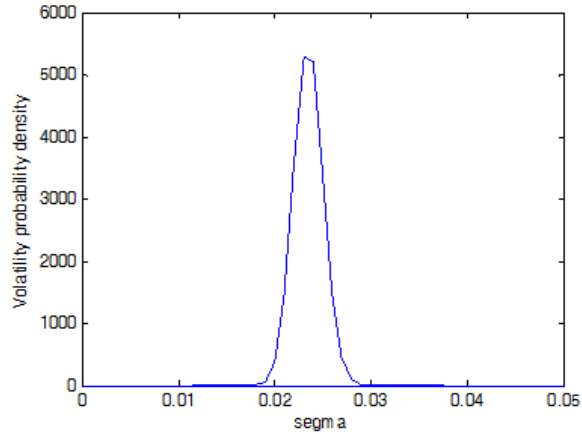

(b)

Fig. 5. JCI variance and volatility probability density

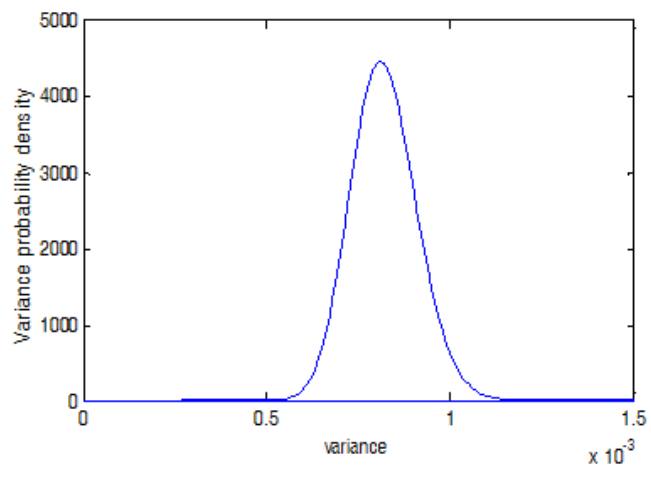

(a)

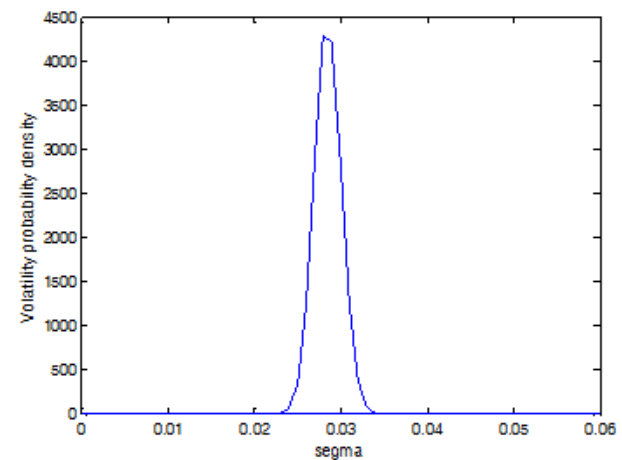

(b)

Fig. 6. LQ45 variance and volatility probability density

Each index has different constant parameters even though the indexes follow the same diffusion process. Moreover, each index has different fluctuation. The probability density of variance and volatility for each index at stationary state is presented on Fig. 5-7, determined by making use of Equation 11 with parameters given in Table 1 .

The probability density of variance in Fig. 5-7 reach their maximum value at $\eta$. It means that the distribution was maximum at long time variance. 


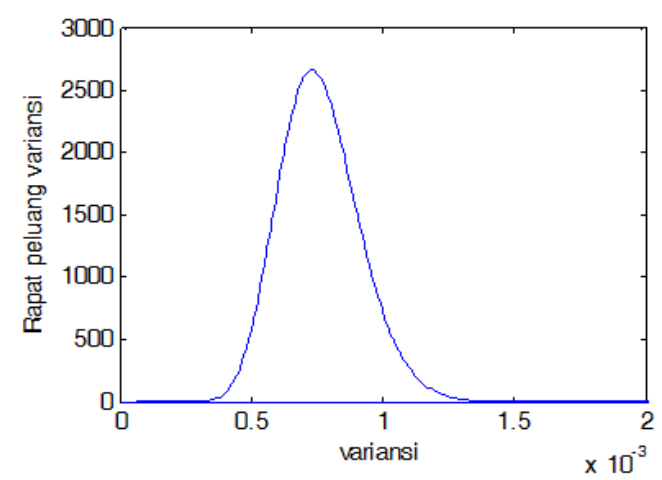

(a)

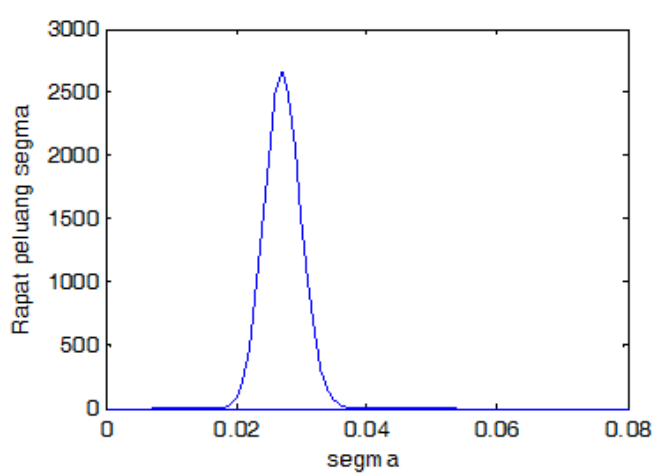

(b)

Fig. 7. JII variance and volatility probability density

\section{Conclusion}

The theoretical return probability density is in agreement with the empirical return probability density obtained from Indonesian Stock Exchange (IDX) data series. The theoretical return probability density with stochastic variance is closer to the empirical one than that of the Gaussian, especially at the tail of return probability density. Both the theoretical and the empirical return probability density have Gaussian distribution at peak, but the tail of both probability density is fatter than that of the Gaussian. The tail of each empirical return probability density is closer to that of our theoretical return probability density than that of the Gaussian distribution. The empirical (and the theoretical) return probability densities are not symmetric as in the Gaussian distribution. This theoretical stochastic variance model is more comparable to data series than that of the Gaussian.

The variance of the stock price in Indonesian Stock Exchange IDX follows Ornstein-Uhlenbeck process. The variance probability density at stationary state can be obtained by fitting the empirical probability density obtained from Indonesian Stock Exchange IDX data series with an integral expression obtained from the quantum mechanical method.

\section{Author's Contributions}

Dwi Satya Palupi: Is the main research of project. She conducted the theoretical calculation and providing the initial draft of the manuscript.

Eduardus Tandelilin: Is the second supervisor (copromotor) for D.S. Palupi and over saw the analyzed of the theoretic probability density and reviewed the content of the manuscript.

Arief Hermanto: Is the third supervisor (co-promotor) for Dwi Satya Palupi and oversaw the theoretical calculation and reviewed the content of the manuscript.
Muhammad Farchani Rosyid: Is the first supervisor (promotor) for Dwi Satya Palupi who provided the main idea and oversaw the overall research project as well as reviewing the manuscript.

\section{Ethics}

This article is original and to the best knowledge of the authors has not been published before. The authors confirm that there are no ethical issues involved.

\section{Reference}

Belal, B.E., 2004. Quantum Finance: Path Integrals and Hamiltonians for Options and Interest Rates. 1st Edn., Cambridge University Press, New York, ISBN-10: 0521714788, pp: 336.

Black, F. and M. Scholes, 1973. The pricing of option and corporate liabilities. J. Polit. Economy, 81: 637-654. DOI: $10.1086 / 260062$

Carranco, S.M.G., J.B. Reyes and A.S. Balankin, 2016. The crude oil price bubbing and universal scaling dynamic of price volatility. Phys. A, 452: 60-68. DOI: $10.1016 /$ j.physica.2016.02.006

Dragulescu, A.A. and V.M. Yakovenko, 2002. Probability distribution of returns in the Heston model with stochastic volatility. Quant. Finan., 2: 443-453. DOI: 10.1080/14697688.202.000011

Guhathakurta, K., I. Mukherjee and A.R. Chowdhury, 2008. Empirical mode decomposition analysis of two different financial time series and their comparison. Chaos, Soliton Fractal, 37: 1214-1227. DOI: 10.1016/j.chaos.2006.10.065

Huang, N.E., M.L. Wu, W. Qu, S.R. Long and S.S.P. Shen, 2003. Application of Hilbert-Huang transform to non-stationary financial time series analysis. Applied Stochastic Models Bus. Ind., 19: 245-268. DOI: 10.1002/asmb.501

Hull, J. and A. White, 1987. The pricing of Options on assets with stochastic volatilities. J. Finance, 42: 281-300. DOI: 10.1111/j.1540-6261.1987.tb02568.x 
Heston, S.L., 1993. A closed-form solution for option with stochastic volatility wit application to bond currency option. Rev. Finan. Stud., 6: 327-343. DOI: $10.1093 / \mathrm{rfs} / 6.2 .327$

Kuo, H.H., 2006. Introduction to Stochastic Integration. 1st Edn., Springer Science and Business Media, New York, ISBN-10: 0387310576, pp: 279.

Mandelbrot, B., 1963. The variation of certain speculative price. J. Bus., 36: 394-419. PMID: 2350970

Mantegna, R.N. and H.E. Stanley, 2000. An introduction to econophysics: Correlations and complexity in finance. Phys. Today, 53: 70-70. DOI: $10.1063 / 1.1341926$

Nava, N., TD. Matteo and T. Aste, 2016. Anomalous Volatility Scaling in high frequency financial data. Phys. A, 447: 434-445.

DOI: $10.1016 /$ j.physa.2015.12.022
Palupi, D.S., A. Hermanto, E. Tandelilin and M.F. Rosyid, 2014. The application of path integral for $\log$ return probability calculation. J. Phys. Conf. Series, 539: 012017-012017.

DOI: $10.1088 / 1742-6596 / 539 / 1 / 01 / 01217$

Stein, E.M. and J.C. dan Stein, 1991. Stock price distribution with stochastic volatility: An analytic approach. Rev. Finan. Stud., 4: 727-752. DOI: $10.1093 / \mathrm{rfs} / 4.4 .727$

Yamasaki, K., L. Muchnik, S. Havlin, A. Bunde and H.E. Stanley, 2005. Scaling and memory in volatility return intervals in financial markets. Proc. Natl. Acad. Sci. USA, 102: 9424-9428. DOI: $10.1073 /$ pnas.0502613102 